\title{
Special Care Units and Traditional Care in Dementia: Relationship with Behavior, Cognition, Functional Status and Quality of Life - A Review
}

\author{
Jeroen S. Kok ${ }^{a}$ Ina J. Berg ${ }^{a}$ Erik J.A. Scherder ${ }^{b}$ \\ a Lentis/Dignis, Mental Health Care Institute, Zuidlaren, and bepartment of \\ Clinical Neuropsychology, VU University Amsterdam, Amsterdam, The Netherlands
}

\section{Key Words}

Dementia - Nursing home - Special care units - Behavior - Cognition · Functional status .

Quality of life

\begin{abstract}
Background: Special care facilities for patients with dementia gain increasing attention. However, an overview of studies examining the differences between care facilities with respect to their effects on behavior, cognition, functional status and quality of life is lacking. Results: Our literature search resulted in 32 studies published until October 2012. Overall, patients with dementia who lived at special care units (SCUs) showed a significantly more challenging behavior, more agitation/aggression, more depression and anxiety, more cases of global cognitive impairment and a better psychosocial functioning. There was a tendency towards a better functional status in specialized care facilities, and a better quality of life was found in favor of the SCU group compared to the traditional nursing home (n-SCU) group. Longitudinal studies showed an increased number of neuropsychiatric cases, more patients displaying deteriorating behavior and resistance to care as well as less decline in activities of daily living $(A D L)$ in the SCU group compared to the $n-S C U$ group. Patients in small-scale, homelike SCUs showed more agitation and less ADL decline compared to SCU patients. Conclusion: This review shows that the patient characteristics in SCU and n-SCU settings and, to a minor extent, in SCU and small-scale, homelike SCU settings are different. Over time, there are differences between n-SCU, SCU and small-scale, homelike SCU facilities for some variables.
\end{abstract}


Kok et al.: Special Care Units and Traditional Care in Dementia: Relationship with Behavior, Cognition, Functional Status and Quality of Life - A Review

\section{Introduction}

There are different types of care facilities for elderly subjects suffering from dementia. There is a general view that special care units (SCUs) with trained personnel are the most appropriate environment to enhance the quality of care for subjects who suffer from dementia $[1,2]$. In the Netherlands, SCUs or small-scale, homelike SCUs are currently the most common types of living environments for patients with dementia who cannot live on their own anymore [3]. SCUs have arisen with the development of dementia care in addition to the more traditional medical approach. Traditionally, patients with dementia reside in institutions that follow a medical-somatic approach without specialization of dementia care $[4,5]$. However, there is no final definition for SCUs. They are especially designed for patients with dementia, implying resident security and safety through locking systems, signposts and communal living areas [6]. The staff is specially trained to deal with behavioral and psychological symptoms of dementia (BPSD), a heterogeneous collection of behaviors and noncognitive symptoms occurring in the course of dementia [7]. This specialization results in a better organized care offered by SCUs [8] compared to traditional nursing homes (n-SCUs) and in more family involvement [9]. Besides SCUs with large wards, also small-scale, homelike SCUs with 8-12 patients in one unit are offered to patients with dementia. Care providers in these units follow a vision of long-term care by emphasizing normalization of daily life [10-15].

The general purpose of SCUs is a non-drug treatment of dementia symptoms [16,17], that is to reduce the behavioral and psychological problems associated with dementia [18-22]. An SCU unit can further be divided into separate sections, e.g. for patients with severe agitation and disruptive behavior and for those with mild BPSD [7].

The physical environment has been recognized as an influential element in the care of dementia patients [23]. For example, it has been demonstrated that dementia patients with more privacy express less anxiety and aggression [19]. In another study, patients of a large, $\mathrm{n}$-SCU showed higher levels of aggression than those living in the less institutionalized settings of SCUs [21].

An n-SCU is mostly defined as a care facility that follows a traditional medical model and does not provide special facilities for dementia patients or specific training for the personnel. The differences between SCUs and n-SCUs are not always obvious. The most evident difference is that training on the job takes place more often at SCUs than at n-SCUs [24]. In a study in which the settings were different but the quality of care was similar, it was demonstrated that patients in SCUs showed different outcome characteristics, namely a greater decline in functional status, expressive language skills and social skills than those in n-SCUs [25]. However, emotional behavior at baseline seemed to influence all outcomes except functional status. Better affect positively influenced the outcome characteristics regardless of the type of setting [25]. Furthermore, it seems that the patient characteristics between SCUs and n-SCUs can be distinct due to differences in patient allocation [26].

There is no international overview comparing care facilities for patients with dementia in consideration of the variables behavior (BPSD), cognition, functional status and quality of life. An analysis of these variables can help clinicians and policy makers to improve the care for patients with dementia in the future. Based on the above-mentioned studies, the present review addresses the question whether the characteristics of dementia patients living in small-scale, homelike SCUs or SCUs are different from those of patients living in SCUs or n-SCUs. 
Kok et al.: Special Care Units and Traditional Care in Dementia: Relationship with Behavior, Cognition, Functional Status and Quality of Life - A Review

\section{Methods}

\section{Search Procedure}

The search focussed on studies published in English from January 1990 until October 2012. All studies providing data on n-SCUs in comparison with SCUs or small-scale, homelike SCUs and SCUs in comparison with small-scale, homelike SCUs for patients with dementia were included in the review.

All combinations of the key words small-scale living, homelike living, group size, traditional nursing home, neuropsychiatric symptoms, behavio(u)r, dementia, cognition and special care unit(s) were entered in four databases Pubmed, SocINDEX, PsychINFO and Ovid. Because of the large amount of articles found after the first search, we present only the relevant ones.

First, titles and abstracts were searched for comparisons between different living situations of patients with dementia. The full text was retrieved when it might be relevant. Twentythree articles met the inclusion criteria. Subsequently, the reference lists of retrieved studies were searched for additional articles. A selection was made among these possibly relevant studies, and the abstracts were browsed in an electronic database as described above. This strategy yielded 6 additional papers. Finally, a further 3 studies $[25,27,28]$ well-known to the authors were also added to the review.

In total, 32 studies were included in the review. Seventeen of these were cross-sectional studies and 15 longitudinal ones. For the longitudinal studies, the baseline scores were also used to calculate the effect sizes to explore the differences in patient characteristics between the populations studied. All baseline scores in the longitudinal studies were not matched and therefore useful as cross-sectional information for the review.

The majority of the studies (25 of 32) were n-SCU/SCU comparisons. Furthermore, 3 n-SCU/small-scale, homelike SCU [14, 15, 29] and 4 SCU/small-scale, homelike SCU [10-13] comparisons were reviewed. These differences are specified later in the text. Only research publications were included in the literature search.

\section{Selection Criteria}

Publications were included when they compared an n-SCU with an SCU or small-scale, homelike SCU, or an SCU with a small-scale, homelike SCU for patients with dementia. The second criterion was that $\mathrm{p}$ values, effect sizes and/or numbers of participants, mean scores and standard deviations had to be available for at least one of the following variables: behavior, cognition, functional status or quality of life. Studies that did not meet these criteria were excluded from the review.

\section{Data Analysis}

The scores on behavior, cognition, functional status and quality of life of patients with dementia in two different care situations were collected. The effect sizes (Cohen's d) were calculated based on the numbers of participants, mean scores and standard deviations. First, the pooled standard deviation was calculated followed by Cohen's d. The baseline data were used to calculate the effect sizes for the longitudinal studies in order to compare them with the cross-sectional research papers. (For an overview of the research design of the included studies and the p values available from the reviewed articles, see tables 1-4.)

None of the papers relevant in substance was excluded because of missing data, and only studies already published were used for the review. In line with Cohen [30], we considered an effect size of $0.0-0.1$ to be nil, one of $0.11-0.2$ to be very small, one of $0.21-0.5$ to be small, one of $0.51-0.8$ to be moderate and one of $\geq 0.81$ to be large. 


\section{Results}

The studies are summarized in four tables, presenting consecutively the variables BPSD (table 1), cognition (table 2), functional status/activities of daily living (ADL) (table 3) and quality of life (table 4) in descending order of the year of publication. The tables present the type of study, the numbers of participants, the specific variables, the assessment scales used, $\mathrm{p}$ values and effect sizes. In the first part of the Results section, the baseline data are presented, followed by the results of the longitudinal data. The studies comparing n-SCUs or SCUs with small-scale, homelike SCUs are denoted in the text and tables.

Cross-Sectional Information: Behavioral and Psychological Symptoms of Dementia

BPSD were divided into global behavior, agitation and aggression, social functioning, depression and anxiety and other behavioral aspects.

\section{Global Behavior}

In total, 18 studies with cross-sectional information about behavioral functioning in the two settings were available (table 1). A global behavioral score at baseline, mostly labelled as 'neuropsychiatry' or 'behavioral problems', was given in 8 studies. In 3 of them [12, 31, 32], the calculated effect sizes were nil.

In 5 studies, large [33], moderate [9, 34, 35] and small [20,36] effect sizes with a more challenging behavior in SCUs as compared to n-SCUs were found.

\section{Agitation and Aggression}

In 3 studies, the effect sizes were nil $[12,21,37]$, and in 1 paper, no significant $p$ value was found [14] for agitation/aggression between SCUs and n-SCUs at baseline.

In 2 surveys [31, 34], a small effect size was calculated, reflecting more agitation in the SCU group. However, in 1 of these studies [31], after matching the patients according to gender, mobility and noncognitive symptoms, the difference in agitation between both groups was not significant anymore. In another study, a significant $\mathrm{p}$ value indicating more agitation / aggression in the SCU group was found [26]. One study showed more aggression in the n-SCU group, with a small effect size [28].

\section{Social Functioning}

Aspects of social functioning were examined in 5 studies. In 1 of them, the calculated effect size at baseline was nil for social activities such as going to the barber and social withdrawal [32], and in another paper, no significant $p$ value was found for participation in pleasant events and social withdrawal [14]. Two studies showed large [38] or small [20] effect sizes, implying that patients living in the SCU showed a better psychosocial function compared to n-SCU patients. One study [15] showed a moderate effect size, indicating a distinct psychosocial behavior in a small patient population from a small-scale, homelike SCU compared to patients from an n-SCU.

\section{Depression and Anxiety}

Depressive behavior was investigated in 7 studies. In 4 of them, a nil effect size $[32,38]$ or a significant $p$ value $[26,39]$ were found. Three studies showed a large [34] and a small $[20,28]$ effect size, suggesting more depressive symptoms in the SCU population at baseline.

In 4 studies, anxiety was assessed. In 2 studies, no significant $\mathrm{p}$ value was found between an n-SCU and a small-scale, homelike SCU [14] and between an n-SCU and an SCU [39]. In 2 other studies, a small effect size [34] and a significant $p$ value were found [26], indicating more anxiety in the SCU group compared to the n-SCU group. 


DOI: $10.1159 / 000353441$

(c) 2013 S. Karger AG, Basel www.karger.com/dee

Kok et al.: Special Care Units and Traditional Care in Dementia: Relationship with Behavior, Cognition, Functional Status and Quality of Life - A Review

Table 1. BPSD, effect sizes and p values

\begin{tabular}{|c|c|c|c|c|c|c|}
\hline First author & Design & $\begin{array}{l}\text { SCU vs. } \\
\text { n-SCU, n }\end{array}$ & Results & $\begin{array}{l}\text { Assessment } \\
\text { instrument }\end{array}$ & $\mathrm{p}$ value & $\begin{array}{l}\text { Cohen's d } \\
\text { (baseline) }\end{array}$ \\
\hline De Rooij [10] & $\begin{array}{l}\text { quasi-experimental, } \\
\text { longitudinal }^{\mathrm{d}}\end{array}$ & $\begin{array}{l}51 \text { vs. } 51 \\
30 \text { vs. } 47\end{array}$ & $\begin{array}{l}\text { - social engagement } \\
\text { - depression } \\
\text { - behavioral problems } \\
\text { - social engagement } \\
\text { - depression } \\
\text { - behavioral problems }\end{array}$ & NPI [62] & $\begin{array}{l}<0.01^{\mathrm{a}} \\
\text { n.s. } \\
\text { n.s. } \\
\text { n.s. } \\
<0.10^{\text {a }} \\
\text { n.s. }\end{array}$ & $\begin{array}{l}\text { not } \\
\text { available }\end{array}$ \\
\hline $\begin{array}{l}\text { Abrahamson } \\
\text { [39] }\end{array}$ & $\begin{array}{l}\text { interviews, random, } \\
\text { cross-sectional }\end{array}$ & $\begin{array}{l}665 \text { vs. } \\
12,442\end{array}$ & $\begin{array}{l}\text { - depression } \\
\text { - anxiety }\end{array}$ & MDS [63] & $\begin{array}{l}0.743 \\
0.029\end{array}$ & $\begin{array}{l}\text { not } \\
\text { available }\end{array}$ \\
\hline Nazir [42] & cohort study & $\begin{array}{l}2,843 \text { vs. } \\
23,322\end{array}$ & $\begin{array}{l}\text { - worsening behavior } \\
\text { - more verbally abusive } \\
\text { - more physically abusive } \\
\text { - more socially } \\
\text { inappropriate } \\
\text { - growing resistance to care }\end{array}$ & MDS & $\begin{array}{r}<0.001^{\mathrm{a}} \\
0.399^{\mathrm{a}} \\
0.049^{\mathrm{a}} \\
0.063^{\mathrm{a}} \\
<0.001^{\mathrm{a}}\end{array}$ & $\begin{array}{l}\text { not } \\
\text { available }\end{array}$ \\
\hline Verbeek [12] & $\begin{array}{l}\text { quasi-experimental, } \\
\text { longitudinal, controlled }\end{array}$ & $\begin{array}{l}124 \text { vs. } \\
135\end{array}$ & $\begin{array}{l}\text { - neuropsychiatry } \\
\text { - agitation }\end{array}$ & $\begin{array}{l}\text { NPI } \\
\text { CMAI-D [64] }\end{array}$ & $\begin{array}{l}\text { n.s. } \\
0.035^{\mathrm{a}}\end{array}$ & $\begin{array}{r}0.04 \\
-0.02\end{array}$ \\
\hline Weyerer [31] & $\begin{array}{l}\text { cross-sectional, randomly } \\
\text { selected, matched }\end{array}$ & $\begin{array}{l}594 \text { vs. } \\
573\end{array}$ & $\begin{array}{l}\text { - neuropsychiatry } \\
\text { - agitation }\end{array}$ & $\begin{array}{l}\text { NPI } \\
\text { CMAI [65] }\end{array}$ & $\begin{array}{l}\text { n.s. } \\
<0.001\end{array}$ & $\begin{array}{l}0.07 \\
0.38\end{array}$ \\
\hline $\begin{array}{l}\text { Te Boekhorst } \\
\text { [13] }\end{array}$ & $\begin{array}{l}\text { quasi-experimental, } \\
\text { longitudinal, matched, } \\
\text { controlled }^{\mathrm{d}}\end{array}$ & 67 vs. 97 & $\begin{array}{l}\text { - depression } \\
\text { - behavior } \\
\text { - neuropsychiatry } \\
\text { - social engagement }\end{array}$ & $\begin{array}{l}\text { RMBPC [66] } \\
\text { NPI } \\
\text { RISE [67] }\end{array}$ & $\begin{array}{l}\text { n.s. }{ }^{\text {a }} \\
\text { n.s. } \\
\text { n.s. } \\
<0.05^{\text {a }}\end{array}$ & $\begin{array}{l}\text { not } \\
\text { available }\end{array}$ \\
\hline Selbaek [26] & cross-sectional & $\begin{array}{l}313 \text { vs. } \\
762\end{array}$ & $\begin{array}{l}\text { - delusions } \\
\text { - hallucination } \\
\text { - depression } \\
\text { - anxiety } \\
\text { - euphoria } \\
\text { - agression/agitation } \\
\text { - apathy } \\
\text { - disinhibition } \\
\text { - aberrant motor behavior }\end{array}$ & NPI & $\begin{array}{l}\leq 0.001 \\
\leq 0.001 \\
\text { n.s. } \\
\leq 0.01 \\
\leq 0.001 \\
\leq 0.001 \\
\text { n.s. } \\
\text { n.s. } \\
\leq 0.01\end{array}$ & $\begin{array}{l}\text { not } \\
\text { available }\end{array}$ \\
\hline Nobili [9] & longitudinal comparative & 72 vs. 72 & - neuropsychiatry & NPI & $0.0001^{\mathrm{a}}$ & -0.74 \\
\hline Pekkarinen [33] & cross-sectional & $\begin{array}{l}390 \text { vs. } \\
587\end{array}$ & - behavioral problems & LRAI [68] & $<0.001$ & 1.16 \\
\hline Morgan [37] & experimental, cross-sectional & $\begin{array}{l}186 \text { vs. } \\
169\end{array}$ & $\begin{array}{l}\text { - exposure to disruptive } \\
\text { behavior } \\
\text { - exposure to aggression }\end{array}$ & $\begin{array}{l}\text { EDB [69] } \\
\text { EAC [69] }\end{array}$ & $\begin{array}{l}<0.01 \\
<0.05\end{array}$ & $\begin{array}{l}-0.028 \\
-0.027\end{array}$ \\
\hline Sloane [32] & cross-sectional, random & $\begin{array}{l}773 \text { vs. } \\
479\end{array}$ & $\begin{array}{l}\text { - behavioral problems } \\
\text { - depressive symptom } \\
\text { - social functioning } \\
\text { - social withdrawal }\end{array}$ & $\begin{array}{l}\text { CMAI } \\
\text { Cornell [70] } \\
\text { no standardized } \\
\text { instrument }{ }^{\mathrm{b}} \\
\text { MOSES [71] }\end{array}$ & $\begin{array}{l}\text { n.s. } \\
0.001 \\
0.001 \\
0.001\end{array}$ & $\begin{array}{r}-0.001 \\
0.007 \\
0.012 \\
-0.015\end{array}$ \\
\hline Reimer [14] & matched groups ${ }^{\mathrm{e}}$ & 62 vs. 59 & $\begin{array}{l}\text { - agitation } \\
\text { - social withdrawal } \\
\text { - affect (anxiety) } \\
\text { - socially appropriate } \\
\text { behavior }\end{array}$ & $\begin{array}{l}\text { CMAI } \\
\text { MOSES } \\
\text { AARS [72] } \\
\text { Pleasant Events } \\
\text { scale [73] }\end{array}$ & $\begin{array}{l}\text { n.s. } \\
\text { n.s. } \\
\text { n.s. } \\
\text { n.s. }\end{array}$ & $\begin{array}{l}\text { not } \\
\text { available }\end{array}$ \\
\hline Warren [38] & longitudinal, controlled & 44 vs. 36 & $\begin{array}{l}\text { - depression } \\
\text { - psychosocial functioning }\end{array}$ & $\begin{array}{l}\text { Cornell } \\
\text { MOSES }\end{array}$ & $\begin{array}{l}\text { not } \\
\text { available }\end{array}$ & $\begin{array}{r}-0.06 \\
1.82\end{array}$ \\
\hline Chappel [25] & $\begin{array}{l}\text { experimental, longitudinal, } \\
\text { controlled }\end{array}$ & total: 323 & $\begin{array}{l}\text { - agitation } \\
\text { - social skills } \\
\text { - affect }\end{array}$ & $\begin{array}{l}\text { CMAI } \\
\text { MAS-R [74] } \\
\text { FTQ }\end{array}$ & $\begin{array}{c}\text { n.s. }{ }^{\text {a }} \\
<0.05^{\text {a }} \\
\text { n.s. }\end{array}$ & $\begin{array}{l}\text { not } \\
\text { available }\end{array}$ \\
\hline Leon [21] & experimental field study & $\begin{array}{l}432 \text { vs. } \\
164\end{array}$ & $\begin{array}{l}\text { - aggressive behavior } \\
\text { - disruptive behavior }\end{array}$ & $\begin{array}{l}\text { CMAI } \\
\text { MDS }\end{array}$ & $\begin{array}{c}\text { n.s. } \\
<0.01\end{array}$ & $\begin{array}{l}0.05 \\
0.24\end{array}$ \\
\hline
\end{tabular}


Table 1 (continued)

\begin{tabular}{|c|c|c|c|c|c|c|}
\hline First author & Design & $\begin{array}{l}\text { SCU vs. } \\
\text { n-SCU, n }\end{array}$ & Results & $\begin{array}{l}\text { Assessment } \\
\text { instrument }\end{array}$ & $\mathrm{p}$ value & $\begin{array}{l}\text { Cohen's d } \\
\text { (baseline) }\end{array}$ \\
\hline Frisoni [34] & longitudinal, controlled & 31 vs. 35 & $\begin{array}{l}\text { - delusions } \\
\text { - hallucinations } \\
\text { - agitation } \\
\text { - anxiety } \\
\text { - euphoria/elation } \\
\text { - disinhibition } \\
\text { - irritability/lability } \\
\text { - abberant motor behavior } \\
\text { - sleep } \\
\text { - total neuropsychiatry } \\
\text { - agitation } \\
\text { - depression }\end{array}$ & $\begin{array}{l}\text { CMAI } \\
\text { Cornell }\end{array}$ & $\begin{array}{l}\text { not } \\
\text { available }\end{array}$ & $\begin{array}{r}0.33 \\
0.44 \\
0.46 \\
0.27 \\
-0.07 \\
0.12 \\
0.42 \\
0.18 \\
0.20 \\
0.64 \\
0.49 \\
1.05\end{array}$ \\
\hline Saxton [15] & $\begin{array}{l}\text { longitudinal, matched, } \\
\text { controlled }^{\mathrm{e}}\end{array}$ & 26 vs. 19 & - social/cognitive & NHBPS [75] & n.s. ${ }^{a}$ & 0.56 \\
\hline Kovach [36] & behavioral observations & 23 vs. 14 & - functional behavior & $\begin{array}{l}\text { no standardized } \\
\text { instrument }^{\mathrm{c}}\end{array}$ & $\begin{array}{l}\text { not } \\
\text { available }\end{array}$ & -0.50 \\
\hline Swanson [35] & $\begin{array}{l}\text { quasi-experimental, } \\
\text { longitudinal }\end{array}$ & 13 vs. 9 & - noncognitive behavior & ADAS [76] & n.s. ${ }^{a}$ & 0.65 \\
\hline Lindesay [28] & cross-sectional $^{\mathrm{f}}$ & 27 vs. 29 & $\begin{array}{l}\text { - depression } \\
\text { - activity disturbance } \\
\text { - aggressivity }\end{array}$ & $\begin{array}{l}\text { DSS [77] } \\
\text { ABRS [78] }\end{array}$ & $\begin{array}{l}\text { not } \\
\text { available }\end{array}$ & $\begin{array}{r}0.11 \\
0.28 \\
-0.21\end{array}$ \\
\hline Chafetz [46] & $\begin{array}{l}\text { quasi-experimental, } \\
\text { longitudinal }\end{array}$ & 12 vs. 8 & - behavior & BRF [79] & n.s. ${ }^{a}$ & $\begin{array}{l}\text { not } \\
\text { available }\end{array}$ \\
\hline Holmes [20] & $\begin{array}{l}\text { quasi-experimental, } \\
\text { longitudinal }\end{array}$ & 49 vs. 44 & $\begin{array}{l}\text { - disturbing behavior total } \\
\text { score } \\
\text { - depression } \\
\text { - social activities }\end{array}$ & INCARE [80] & $\begin{array}{l}\text { n.s. }{ }^{b} \\
\text { n.s. } \\
0.01^{b}\end{array}$ & $\begin{array}{r}0.47 \\
0.36 \\
-0.36\end{array}$ \\
\hline
\end{tabular}

MDS = Minimum data set 2.0 section E4; NPI = Neuropsychiatric Inventory; CMAI(-D) = Cohen-Mansfield Agitation Inventory (-Dutch version); RMBPC = Revised Memory and Behaviour Problems Checklist; RISE $=$ Revised Index of Social Engagement from the Resident Assessment Instrument (RAI); LRAI = Long-Term Care Resident Assessment Instrument; EDB = Exposure to Disruptive Behaviours subscale; EAC $=$ Exposure to Aggression during Caregiving subscale; Cornell $=$ Cornell Scale for Depression in Dementia; MOSES $=$ Multidimensional Observation Scale for Elderly Subjects; AARS = Apparent Affect Rating Scale; MAS-R = Multifocus Assessment Scale-Revised; FTQ = Feeling Tone Questionnaire (no statistics available on research initiation); NHBPS = Nursing Home Behavioral Problem Scale; ADAS = Alzheimer's Disease Assessment Scale; DSS = Depressive Signs Scale; ABRS = Adaptive Behaviour Rating Scale; BRF = Behaviour Rating Form; INCARE = Institutional Comprehensive Assessment and Referral Evaluation; n.s. = not significant.

${ }^{a}$ Over time; ${ }^{b} 17$ items of participation in social activities; ${ }^{c}$ checklist for behavioural mapping in long-term care facilities; ${ }^{\mathrm{d}}$ small-scale, homelike SCU/SCU comparison; ${ }^{\mathrm{e}}$ small-scale, homelike SCU/n-SCU comparison; ${ }^{\mathrm{f}}$ SCU/n-SCU with mixed-sex population.

\section{Other Behavioral Aspects}

Deviant behavior such as delusions, hallucinations, euphoria, apathy, disinhibition and aberrant motor behavior were significantly more prevalent in the SCU group compared to the n-SCU group [26]. One study found more delusions, hallucinations and irritability/lability with small effect sizes and more disinhibition, aberrant motor behavior and sleep disturbances with very small effect sizes in the SCU group compared to the n-SCU group [34]. In this study, no differences were found for euphoria. Activity disturbance with a small effect size was more common in another study [28]. Yet another study showed a nil effect size between both groups in disruptive behavior directed at the nursing staff [18].

Taken together, SCU patients generally showed a more challenging and deviant behavior, more agitation/aggression, more depression and possibly more anxiety. On the other hand, patients in SCUs also showed better psychosocial function compared to those in n-SCUs. Little evidence was found for a distinct psychosocial behavior in the small-scale, homelike SCU compared to the n-SCU. 
Kok et al.: Special Care Units and Traditional Care in Dementia: Relationship with Behavior, Cognition, Functional Status and Quality of Life - A Review

\section{Cognition}

Baseline data on differences between SCU and n-SCU patients that were obtained either with the Mini-Mental State Examination (MMSE) or with a related global cognitive measure were collected by 20 studies (table 2). In 5 studies, a nil effect size [12, 15, 32, 40, 41] was calculated, indicating that both investigated groups were equally cognitively impaired at baseline.

A greater global cognitive impairment in the SCU group was found in 10 studies with large [33, 38, 39, 42], moderate [20, 28, 36] and small effect sizes [21, 34] or significant p values [43]. In contrast, 1 study showed an increased cognitive impairment with a large effect size in a limited number of participants [35], and 1 study showed a moderate effect size in a younger n-SCU patient group [9]. A large effect size for better communication and recognition and a small effect size for better orientation in the SCU group compared to the n-SCU group were also found [28]. In other studies, the nil effect size [41] was calculated for speech abilities in the n-SCU/SCU groups, or memory loss was assessed [34].

Better cognitive performances were found in favor of a small-scale, homelike SCU compared to a SCU with a large effect size [11]. Another study [10] showed small but reverse effect sizes in two small-scale, homelike SCU/SCU group comparisons.

Taken together, a more severe global cognitive impairment and better communication in SCUs compared to n-SCUs were found at baseline. Some evidence for better cognitive performances in small-scale SCUs was found as well.

\section{Functional Status}

ADL or functional ability at baseline was measured in 19 studies (table 3). In 4 studies, a nil effect size [21,32, 41] or no significant $p$ value [39] was found for ADL functioning between SCU and n-SCU residents.

Better functional abilities in the SCU group at baseline compared to the n-SCU group were found in 6 studies with large [8], moderate [9] and small [27, 34, 35] effect sizes or significant p values [44]. More specifically, 1 study [28] showed a large effect size for better washing, a moderate effect size for better dressing and small effect sizes for better feeding, toileting and mobility for the SCU group compared to the n-SCU group.

Two studies showed a large [15] effect size in a small patient group, and a significant $p$ value [14] in favor of the small-scale SCU compared to the n-SCU. Three studies showed better ADL function with large [11], moderate [10] and very small [12] effect sizes in favor of the small-scale SCUs compared to the SCUs.

Five studies showed better ADL or functional abilities, e.g. self-care, for the n-SCU group with large [38], small [33] and very small [20,31] effect sizes or p values [43].

In sum, with regard to functional status and ADL, there is a tendency towards better outcomes in functional status/ADL for the SCUs and small-scale, homelike SCUs compared to less specialized care.

\section{Quality of Life and Remaining Variables}

Five studies presented data about aspects of quality of life at baseline (table 4). Two studies showed either a nil effect size [11] or no significant difference for quality of life between SCUs and small-scale, homelike SCUs [13]. In 1 study [29], a small effect size was calculated for the total quality of life in favor of the SCU group compared to the n-SCU patients, whereas the different subscales of the observation list in this study showed small to very small effect sizes in favor of the SCU group. Another study [39] also showed very small effect sizes for comfort and environmental adaption in favor of the SCU group, and no differences for the other quality of life variables. 
Table 2. Cognition, effect sizes and p values

\begin{tabular}{|c|c|c|c|c|c|c|}
\hline First author & Design & $\begin{array}{l}\text { SCU vs. } \\
\text { n-SCU, n }\end{array}$ & Results & $\begin{array}{l}\text { Assessment } \\
\text { instrument }\end{array}$ & $\mathrm{p}$ value & $\begin{array}{l}\text { Cohen's d } \\
\text { (baseline) }\end{array}$ \\
\hline De Rooij [10] & $\begin{array}{l}\text { quasi-experimental, } \\
\text { longitudinalc }^{c}\end{array}$ & $\begin{array}{l}51 \text { vs. } 51 \\
30 \text { vs. } 47\end{array}$ & $\begin{array}{l}\text { - cognition } \\
\text { - cognition }\end{array}$ & MMSE [81] & not available & $\begin{array}{r}-0.45 \\
0.31\end{array}$ \\
\hline $\begin{array}{l}\text { Abrahamson } \\
\text { [39] }\end{array}$ & random, cross-sectional & $\begin{array}{l}665 \text { vs. } \\
12,442\end{array}$ & - cognitive impairment & CPS [82] & $<0.001$ & 1.02 \\
\hline Nazir [42] & cohort study & $\begin{array}{l}2,843 \text { vs. } \\
23,322\end{array}$ & - cognitive impairment & CPS & $<0.001^{\mathrm{a}}$ & 0.96 \\
\hline Verbeek [12] & $\begin{array}{l}\text { quasi-experimental, } \\
\text { longitudinal, controlled }^{c}\end{array}$ & 124 vs. 135 & - cognition & MMSE & n.s. & 0.09 \\
\hline Verbeek [11] & cross-sectional & 586 vs. 183 & - cognition & CPS/MDS [82] & n.s. & 11.76 \\
\hline $\begin{array}{l}\text { Te Boekhorst } \\
{[13]}\end{array}$ & $\begin{array}{l}\text { quasi-experimental, } \\
\text { longitudinal, matched, } \\
\text { controlled }^{c}\end{array}$ & 67 vs. 97 & $\begin{array}{l}\text { - cognitive functioning } \\
\text { - memory }\end{array}$ & $\begin{array}{l}\text { MMSE } \\
\text { RMBPC [66] }\end{array}$ & $\begin{array}{l}\text { n.s. }{ }^{a} \\
\text { n.s. }^{a}\end{array}$ & $\begin{array}{l}\text { not } \\
\text { available }\end{array}$ \\
\hline Nobili [9] & longitudinal, comparative & 72 vs. 72 & - cognitive performance & MMSE & n.s. ${ }^{a}$ & 0.72 \\
\hline $\begin{array}{l}\text { Pekkarinen } \\
\text { [33] }\end{array}$ & $\begin{array}{l}\text { cross-sectional, selection } \\
\text { by characteristics }\end{array}$ & 390 vs. 587 & - cognition & CPS & $<0.001$ & 1.24 \\
\hline Sloane [32] & cross-sectional, random & 773 vs. 479 & - cognitive status & MDS-COGS [82] & 0.005 & -0.007 \\
\hline Warren [38] & longitudinal, controlled & 44 vs. 36 & - cognitive status & MMSE & not available & -1.96 \\
\hline Chappel [25] & experimental & total: 323 & $\begin{array}{l}\text { - cognitive functioning } \\
\text { - expressive language skills }\end{array}$ & MAS-R [74] & $\begin{array}{l}\text { n.s. }{ }^{\text {a }} \\
<0.01^{\text {a }}\end{array}$ & $\begin{array}{l}0.009 \\
0.156\end{array}$ \\
\hline Leon [21] & experimental field study & 432 vs. 164 & - cognitive limitations & MDS-COGS & $<0.001$ & 0.31 \\
\hline McAllister [40] & cross-sectional & 59 vs. 34 & - cognitive functioning & MMSE & not available & 0.01 \\
\hline Frisoni [34] & controlled study & 31 vs. 35 & $\begin{array}{l}\text { - cognitive status } \\
\text { - memory loss }\end{array}$ & $\begin{array}{l}\text { MMSE } \\
\text { CDR [83] }\end{array}$ & $\begin{array}{l}\text { n.s. } \\
\text { n.s. }\end{array}$ & $\begin{array}{r}-0.20 \\
0.03\end{array}$ \\
\hline Saxton [15] & $\begin{array}{l}\text { longitudinal, matched, } \\
\text { controlled }^{\mathrm{d}}\end{array}$ & 26 vs. 19 & - mental status & MMSE & n.s. ${ }^{\mathrm{a}}$ & -0.04 \\
\hline Kovach [36] & behavioral observations/tests & 23 vs. 14 & - mental status & MMSE & not available & 0.52 \\
\hline Volicer [41] & prospective cohort study & 50 vs. 112 & $\begin{array}{l}\text { - cognitive impairment } \\
\text { - speech }\end{array}$ & $\begin{array}{l}\text { MMSE } \\
\text { BADE [84] }\end{array}$ & $\begin{array}{l}<0.05 \\
\text { n.s. }\end{array}$ & $\begin{array}{r}-0.10 \\
0.05\end{array}$ \\
\hline Swanson [35] & $\begin{array}{l}\text { quasi-experimental design, } \\
\text { pre-/post-tests }\end{array}$ & 13 vs. 9 & - cognitive behavior & ADAS [76] & n.s. ${ }^{\mathrm{a}}$ & 1.12 \\
\hline Lindesay [28] & cross-sectional $^{\mathrm{e}}$ & 27 vs. 29 & $\begin{array}{l}\text { - cognitive impairment } \\
\text { - communication } \\
\text { - recognition } \\
\text { - orientation }\end{array}$ & $\begin{array}{l}\text { OBS [85] } \\
\text { ABRS [78] }\end{array}$ & not available & $\begin{array}{r}-0.67 \\
0.93 \\
1.33 \\
0.29\end{array}$ \\
\hline Chafetz [46] & $\begin{array}{l}\text { quasi-experimental, } \\
\text { longitudinal }\end{array}$ & 12 vs. 8 & - cognitive ability & DRS [86] & n.s. ${ }^{a}$ & $\begin{array}{l}\text { not } \\
\text { available }\end{array}$ \\
\hline Holmes [20] & quasi-experimental & 49 vs. 44 & - dementia scale & K-GMSQ [87] & $0.05^{b}$ & 0.52 \\
\hline Coleman [43] & experimental & 47 vs. 36 & - cognitive decline & RGDS [60] & $<0.01$ & $\begin{array}{l}\text { not } \\
\text { available }\end{array}$ \\
\hline
\end{tabular}

CPS = Cognitive Performance Scale; RMBPC = Revised Memory and Behaviour Problems Checklist; MDS-COGS = Minimum Data Set Cognitive Performance Scale; MAS-R = Multifocus Assessment Scale-Revised; CDR = Clinical Dementia Rating Scale; BADE = Boston Aphasia Diagnostic Evaluation; ADAS = Alzheimer's Disease Assessment Scale; OBS = Organic Brain Syndrome scale; CARE = subscale of the Comprehensive Assessment and Referral Evaluation; ABRS = Adaptive Behaviour Rating Scale; DRS = Mattis Dementia Rating Scale; K-GMSQ = Kahn-Goldfarb Mental Status Questionnaire; RGDS = Reisberg Global Deterioration Scale; n.s. = not significant.

${ }^{a}$ Over time; ${ }^{b}$ follow-up measurement; ${ }^{c}$ small-scale, homelike SCU/SCU comparison; ${ }^{d}$ small-scale, homelike SCU/n-SCU comparison; e SCU/n-SCU with mixed-sex population. 


DOI: $10.1159 / 000353441$

C 2013 S. Karger AG, Base www.karger.com/dee

Kok et al.: Special Care Units and Traditional Care in Dementia: Relationship with Behavior, Cognition, Functional Status and Quality of Life - A Review

Table 3. Functional status/ADL, effect sizes and $p$ values

\begin{tabular}{|c|c|c|c|c|c|c|}
\hline Author & Design & $\begin{array}{l}\text { SCU vs. } \\
\text { n-SCU, n }\end{array}$ & Results & $\begin{array}{l}\text { Assessment } \\
\text { instrument }\end{array}$ & $\mathrm{p}$ value & $\begin{array}{l}\text { Cohen's d } \\
\text { (baseline) }\end{array}$ \\
\hline $\begin{array}{l}\text { Orfaly Cadigan } \\
{[44]}\end{array}$ & longitudinal, controlled & 141 vs. 31 & - functional status & BANS-S [88] & 0.0001 & $\begin{array}{l}\text { not } \\
\text { available }\end{array}$ \\
\hline De Rooij [10] & $\begin{array}{l}\text { quasi-experimental, } \\
\text { longitudinal }^{c}\end{array}$ & $\begin{array}{l}51 \text { vs. } 51 \\
30 \text { vs. } 47\end{array}$ & - functional status & Barthel index [52] & not available & $\begin{array}{l}-0.58 \\
-0.53\end{array}$ \\
\hline $\begin{array}{l}\text { Abrahamson } \\
\text { [39] }\end{array}$ & random, cross-sectional & 665 vs. 12,442 & - functional level & MDS ADL [82] & 0.433 & 0.03 \\
\hline Verbeek [12] & $\begin{array}{l}\text { quasi-experimental, } \\
\text { longitudinal, } \\
\text { controlled }^{\mathrm{c}}\end{array}$ & 124 vs. 135 & $-\mathrm{ADL}$ & ADL-H [82] & n.s. & -0.13 \\
\hline Verbeek [11] & cross-sectional & 586 vs. 183 & - functional status & $\operatorname{MDS}[82]$ & n.s. & 10.72 \\
\hline Weyerer [31] & $\begin{array}{l}\text { cross-sectional, randomly } \\
\text { selected, } \\
\text { matched }\end{array}$ & 594 vs. 573 & $-\mathrm{ADL}$ & Barthel index & $<0.05$ & -0.12 \\
\hline $\begin{array}{l}\text { Te Boekhorst } \\
{[13]}\end{array}$ & $\begin{array}{l}\text { quasi-experimental, } \\
\text { longitudinal, } \\
\text { controlled }^{\mathrm{c}}\end{array}$ & 67 vs. 97 & $-\mathrm{ADL}$ & IDDD [89] & $<0.01^{\mathrm{a}}$ & $\begin{array}{l}\text { not } \\
\text { available }\end{array}$ \\
\hline Nobili [9] & longitudinal, comparative & 72 vs. 72 & - functional status & Barthel index & $0.0005^{\mathrm{a}}$ & 0.56 \\
\hline $\begin{array}{l}\text { Pekkarinen } \\
\text { [33] }\end{array}$ & $\begin{array}{l}\text { cross-sectional, selection } \\
\text { by characteristics }\end{array}$ & 390 vs. 587 & - assistance in ADL & MDS ADL & 0.05 & 0.46 \\
\hline Ashcraft [27] & cross-sectional & 15 vs. 15 & $-\mathrm{ADL}$ & MDS ADL & not available & -0.30 \\
\hline Sloane [32] & cross-sectional, random & 773 vs. 479 & - ADL impairment & MDS ADL & 0.001 & -0.021 \\
\hline Reimer [14] & matched groups design ${ }^{\mathrm{d}}$ & 62 vs. 59 & - functional status & FAST [90] & 0.016 & $\begin{array}{l}\text { not } \\
\text { available }\end{array}$ \\
\hline Luo [8] & cross-sectional & 750 vs. 3,667 & $-\mathrm{ADL}$ & $\begin{array}{l}\text { no standardized } \\
\text { instrument }^{b}\end{array}$ & $>0.01$ & -5.70 \\
\hline Warren [38] & longitudinal, controlled & 44 vs. 36 & - physical status (ADL) & FAM + FIM [59] & not available & -2.30 \\
\hline Chappel [25] & experimental & total: 323 & - physical functioning & MDS ADL & $<0.01$ & $0.176^{\mathrm{c}}$ \\
\hline Leon [21] & experimental field study & 432 vs. 164 & - ADL limitations & MDS ADL & n.s. & 0.07 \\
\hline Frisoni [34] & longitudinal, controlled & 31 vs. 35 & - function & Barthel index & not available & 0.31 \\
\hline Saxton [15] & $\begin{array}{l}\text { longitudinal, matched, } \\
\text { controlled }^{\mathrm{d}}\end{array}$ & 26 vs. 19 & $\begin{array}{l}\text { - total ADL } \\
\text { - self-care }\end{array}$ & FIM & $\begin{array}{l}\text { n.s. }^{\mathrm{a}} \\
<0.05^{\mathrm{a}}\end{array}$ & $\begin{array}{l}5.5 \\
0.11\end{array}$ \\
\hline Phillips [45] & $\begin{array}{l}\text { longitudinal, matched, } \\
\text { controlled }\end{array}$ & $\begin{array}{l}1,228 \text { vs. } 5,904 \\
\text { vs. } 70,205\end{array}$ & - ADL function & MDS ADL & n.s. ${ }^{a}$ & $\begin{array}{l}\text { not } \\
\text { available }\end{array}$ \\
\hline Volicer [41] & prospective cohort study & 50 vs. 112 & $-\mathrm{ADL}$ & Katz ADL index [91] & n.s. & 0.01 \\
\hline Swanson [35] & $\begin{array}{l}\text { quasi-experimental, } \\
\text { pre-/post-tests }\end{array}$ & 13 vs. 9 & $\begin{array}{l}\text { - functional ability } \mathrm{I}^{\mathrm{b}} \\
\text { - functional ability } \mathrm{II}^{\mathrm{b}}\end{array}$ & FAC/ GRS [61] & $\begin{array}{l}\text { n.s.* } \\
\text { n.s.* }\end{array}$ & $\begin{array}{l}0.45 \\
0.03\end{array}$ \\
\hline Lindesay [28] & cross-sectional $^{\mathrm{e}}$ & 27 vs. 29 & $\begin{array}{l}\text { - dressing } \\
\text { - washing } \\
\text { - feeding } \\
\text { - toileting } \\
\text { - mobility }\end{array}$ & ABRS [78] & not available & $\begin{array}{l}0.71 \\
1.02 \\
0.36 \\
0.41 \\
0.32\end{array}$ \\
\hline Chafetz [46] & $\begin{array}{l}\text { quasi-experimental, } \\
\text { longitudinal }\end{array}$ & 12 vs. 8 & $-\mathrm{ADL}$ & Katz ADL index & n.s. ${ }^{\mathrm{a}}$ & $\begin{array}{l}\text { not } \\
\text { available }\end{array}$ \\
\hline
\end{tabular}


Table 3 (continued)

\begin{tabular}{|c|c|c|c|c|c|c|}
\hline Author & Design & $\begin{array}{l}\text { SCU vs. } \\
\text { n-SCU, n }\end{array}$ & Results & $\begin{array}{l}\text { Assessment } \\
\text { instrument }\end{array}$ & $\mathrm{p}$ value & $\begin{array}{l}\text { Cohen's d } \\
\text { (baseline) }\end{array}$ \\
\hline Holmes [20] & quasi-experimental & 49 vs. 44 & $-\mathrm{ADL}$ & Katz ADL index & n.s. ${ }^{b}$ & 0.15 \\
\hline Coleman [43] & experimental & 47 vs. 36 & - ADL functional level & Katz ADL index & $<0.01$ & $\begin{array}{l}\text { not } \\
\text { available }\end{array}$ \\
\hline
\end{tabular}

MDS ADL = Morris scale; IDDD = Interview for the Deterioration of Daily Living Activities in Dementia; ADL-H = MDS; FAC = Functional Ability Checklist; GRS = Assessment Functioning of Geriatric Patients; ABRS = Adaptive Behaviour Rating Scale; BANS-S = Bedford Alzheimer's Nursing Severity-Subscale; n.s. = not significant.

a Over time; ${ }^{b}$ ADLs dependence was measured by the degree of dependence in five ADL (transferring, eating, toileting, dressing, bathing); ' small-scale, homelike SCU/SCU comparison; ${ }^{d}$ small-scale, homelike/n-SCU comparison; ${ }^{\text {e }}$ SCU/n-SCU with mixed-sex population.

Table 4. Quality of life and remaining variables, effect sizes and p values

\begin{tabular}{|c|c|c|c|c|c|c|}
\hline Author & Design & $\begin{array}{l}\text { SCU vs. } \\
\text { n-SCU, n }\end{array}$ & Results & $\begin{array}{l}\text { Assessment } \\
\text { instrument }\end{array}$ & $\mathrm{p}$ value & $\begin{array}{l}\text { Cohen's d } \\
\text { (baseline) }\end{array}$ \\
\hline De Rooij [10] & $\begin{array}{l}\text { quasi-experimental, } \\
\text { longitudinalc }^{c}\end{array}$ & $\begin{array}{l}51 \text { vs. } 51 \\
30 \text { vs. } 47\end{array}$ & $\begin{array}{l}\text { - positive affect } \\
\text { - negative affect } \\
\text { - social relations } \\
\text { - social isolation } \\
\text { - restless behavior } \\
\text { - positive affect } \\
\text { - negative affect } \\
\text { - social relations } \\
\text { - social isolation } \\
\text { - restless behavior }\end{array}$ & QUALIDEM [92] & $\begin{array}{l}<0.001^{\mathrm{a}} \\
\text { n.s. }{ }^{\mathrm{a}} \\
<0.001^{\mathrm{a}} \\
\text { n.s. } \\
\text { n.s. } \\
\text { n.s. }^{\mathrm{a}} \\
\text { n. }^{\mathrm{a}} \\
<0.01^{\mathrm{a}} \\
\text { n.s. } \\
\text { n.s. }^{\mathrm{a}} \\
\text { n.s. } \\
\text { n.a }\end{array}$ & $\begin{array}{l}\text { not } \\
\text { available }\end{array}$ \\
\hline $\begin{array}{l}\text { Abrahamson } \\
\text { [39] }\end{array}$ & $\begin{array}{l}\text { interviews, random, } \\
\text { cross-sectional }\end{array}$ & 665 vs. 12,442 & $\begin{array}{l}\text { - comfort } \\
\text { - activity } \\
\text { - privacy } \\
\text { - environment } \\
\text { - individuality } \\
\text { - autonomy } \\
\text { - relationship } \\
\text { - good mood }\end{array}$ & $\mathrm{NHQL}^{\mathrm{b}}$ [93] & $\begin{array}{r}0.007 \\
0.023 \\
0.198 \\
<0.001 \\
0.495 \\
0.033 \\
0.312 \\
0.007\end{array}$ & $\begin{array}{r}0.11 \\
0.00 \\
-0.06 \\
0.18 \\
-0.03 \\
0.09 \\
-0.01 \\
-0.15\end{array}$ \\
\hline Nakanishi [29] & $\begin{array}{l}\text { experimental, randomized, } \\
\text { cross-sectional }^{\mathrm{d}}\end{array}$ & 616 vs. 750 & $\begin{array}{l}\text { - interacting with } \\
\text { surroundings } \\
\text { - expressing oneself } \\
\text { - experiencing minimum } \\
\text { negative behaviors } \\
\text { - total quality of life }\end{array}$ & QLDJ [94] & $\begin{array}{r}<0.001 \\
<0.001 \\
0.013 \\
<0.001\end{array}$ & $\begin{array}{l}0.28 \\
0.26 \\
0.13 \\
0.34\end{array}$ \\
\hline Verbeek [12] & $\begin{array}{l}\text { quasi-experimental, } \\
\text { cross-sectional, longitudinal }^{\mathrm{c}}\end{array}$ & 124 vs. 135 & - quality of life & QUALIDEM & $0.076^{\mathrm{a}}$ & 0.00 \\
\hline $\begin{array}{l}\text { Te Boekhorst } \\
{[13]}\end{array}$ & $\begin{array}{l}\text { quasi-experimental, } \\
\text { longitudinal, matched, } \\
\text { cross-sectional }^{c}\end{array}$ & 67 vs. 97 & - quality of life & DQoL [95] & n.s. ${ }^{a}$ & $\begin{array}{l}\text { not } \\
\text { available }\end{array}$ \\
\hline Morgan [18] & experimental, cross-sectional & 8 vs. 8 & $\begin{array}{l}\text { - awareness and } \\
\text { orientation } \\
\text { - regulation of stimulation } \\
\text { - continuity of the self }\end{array}$ & PEAP [96] & $\begin{array}{l}<0.05 \\
<0.01 \\
<0.05\end{array}$ & $\begin{array}{l}0.22 \\
0.24 \\
0.49\end{array}$ \\
\hline
\end{tabular}

DQoL = Dementia Quality of Life instrument; PEAP = Professional Environmental Assessment Protocol dimensions; QUALIDEM = Quality of Life Assessment instrument; QLDJ = Quality of Life instrument for Japanese elderly with dementia developed from the Alzheimer's Disease Health-Related Quality of Life (ADRQL); NHQL = Nursing Home Quality of Life scale; n.s. = not significant.

${ }^{a}$ Over time; ${ }^{b}$ domains of the NHQL; ${ }^{c}$ small-scale, homelike SCU/SCU comparison; ${ }^{d}$ small-scale, homelike/n-SCU comparison. 
One research paper also investigated various aspects of quality of life rather than one general outcome measure for quality of life [18]. The investigators studied variables that do not fit in the above-mentioned categories. Small effect sizes were found, implicating ratings on awareness and orientation, regulation of stimulation and continuity of the self that are more positive in SCU residents as compared to n-SCU patients.

Taken together, quality of life is hardly investigated in the field of special care for patients with dementia. Generally, better aspects of quality of life was/were found for the SCU group compared to the n-SCU group. There were no differences between small-scale SCUs and SCUs.

\section{Longitudinal Results}

In 11 studies $[9,10,12,13,15,20,25,35,42,45,46]$, longitudinal results ( $p$ values) for the variables reviewed in this paper are presented. In the sections above, we used the baseline measurements of the studies to compute the effect sizes. However, it is worthwhile to take a closer look at studies with long-term data to address possible effects of staying in an n-SCU, SCU or small-scale, homelike SCU.

'Global behavior' and mood, e.g. depression, did not change significantly over time in 3 n-SCU/SCU studies [20, 35, 46] and 4 small-scale, homelike SCU/SCU studies [10, 12, 13, 15]. On the other hand, neuropsychiatry was much more prevalent in the SCU group compared to the n-SCU group after 18 months [9]. In particular, significantly more cases of deteriorating behavior and resistance to care were observed in the SCU group compared to the n-SCU group over 6 months [42].

More agitation in the SCU group compared to the small-scale, homelike SCU was observed over a period of 12 months [11]. More social engagement and depression were shown in 1 of the 2 investigated small-scale, homelike SCUs [10], while there were no differences in the other. The use of different informants at baseline and 6 months later for all behavioral aspects in a small-scale, homelike SCU/SCU study [13] as well as the baseline differences in an n-SCU/ SCU study [9] complicate the interpretation of the findings of these 2 studies.

With respect to global cognitive functioning, no differences over time were shown in 4 n-SCU/SCU [9, 25, 35, 46] and 2 small-scale, homelike SCU/SCU studies [13, 15]. An increasing cognitive decline over 6 months in SCUs compared to n-SCUs was found in 1 study [42]. A comparable decline on a dementia rating scale for the SCU and n-SCU groups over the same period was also found in another study [20]. Concerning specific cognitive domains, expressive language skills appeared to decline more in the SCU group [25], whereas memory functions did not differ over time between the SCU and the small-scale, homelike SCU groups [13].

Although the majority of the studies showed no differences over time in ADL between SCUs and n-SCUs [20, 35, 45, 46], SCUs and small-scale SCUs [12] or n-SCU and small-scale, homelike SCUs [15], a greater decline in ADL was observed in the SCU group compared to the n-SCU group [25]. A smaller decline has been shown in favor of the SCU group compared to the n-SCU group [9], the small-scale, homelike SCU group compared to the n-SCU patients [15] and the small-scale, homelike SCU group compared to the SCU patients [13]. The latter study used different informants at baseline and 6 months later.

Concerning quality of life, no significant differences in aspects of quality of life could be observed between small-scale, homelike SCUs and SCUs over time [10,12,13]. Aspects of quality of life such as positive affect and social relations were more evident than negative affect in small-scale, homelike SCUs compared to SCUs [10].

Taken together, global behavior and mood did not differ between n-SCUs/SCUs or smallscale, homelike SCUs. Compared to n-SCUs, more neuropsychiatric problems, deteriorating behavior and resistance to care were shown in SCUs. Agitation was observed more in SCUs compared to small-scale, homelike SCUs. Most studies showed no differences in cognition or cognitive decline over time. There is little evidence of a greater decline of expressive language 
Kok et al.: Special Care Units and Traditional Care in Dementia: Relationship with

skills in SCUs compared to n-SCUs. ADL function was mostly not different between the groups studied, and some results were mixed. A smaller decline in ADL was found for SCU patients compared to n-SCU subjects as well as for small-scale, homelike SCU patients compared to SCU residents. For the variable 'quality of life', no differences were found over time. There is some evidence for more positive affect and more social relations in small-scale, homelike SCUs compared to SCUs.

\section{Discussion}

The primary goal of this review was to describe studies that examine possible differences in behavior, cognition, functional status and quality of life of patients either living in a SCU or in an n-SCU. The findings are discussed in more detail below.

The more globally defined behavioral variables showed differences between the investigated groups, with a general tendency toward a more challenging behavior, agitation, depression and anxiety for SCU patients at baseline compared to n-SCU residents. Over time, the differences were not consistent. Dementia patients with more pronounced behavioral problems were most likely to be placed in SCUs [20,21,37]. Indeed, several authors indicated that the allocation of patients prior to the investigation might have caused this difference over time $[9,13,15]$. There is evidence of the positive effects of environmental aspects on the challenging behavior in dementia [47].

The variable 'social functioning' is in favor of the SCU patients at baseline measurements. This discrepancy was not related to differences in cognition at enrolment of the study. The most plausible explanation for this finding is the higher involvement in social activities at SCUs [48].

Specific interventions, e.g. aromatherapy or providing preferred music for nursing home residents with dementia, can produce small to moderate effects on agitated behavior during a short period of time $[49,50]$. These types of interventions occur mostly in SCU facilities because of the specially trained staff [51].

Comparisons of the differences in cognitive functioning between SCU and n-SCU patients show a global tendency towards a greater cognitive impairment in the SCU group at baseline and no differences over time. This does not apply to expressive language skills, which show a greater decline over time in the SCU group [13, 42], indicating differences in the allocation of patients. It has been argued that the course of specific cognitive aspects in dementia can be influenced by different care settings [25].

No clear direction of differences in ADL was found between the groups at baseline and over time. A possible explanation is that the studies looked at different functional abilities, e.g. transferring, eating, toileting, dressing and bathing [8] or levels of disability [38]. Consequently, the investigators used various assessment lists measuring different abilities, e.g. the Barthel index $[31,52]$ or an ADL hierarchy scale $[33,53]$.

Somewhat surprisingly, quality of life has gained minimal attention in the various studies reviewed here. Although it is reasonable to assume that environmental adjustment is only one aspect of the care and treatment of dementia patients, it might have a considerable effect on the subjectively experienced quality of life $[54,55]$. Qualitative research suggests that the quality of life of dementia patients increases in specifically created environments such as SCUs: agitation decreases over time and, according to the staff and relatives, the patients feel free [56]. Furthermore, social interactions between patients show a substantial improvement [57]. However, in this review, not all studies showed a positive effect on quality of life.

Taken together, there seems to be a tendency to assign different patient groups to different care facilities. This holds true particularly for patients living in n-SCUs and SCUs. The 
Kok et al.: Special Care Units and Traditional Care in Dementia: Relationship with

Behavior, Cognition, Functional Status and Quality of Life - A Review

benefit of small-scale SCUs is not convincing compared to 'regular' SCU care. As a whole, this review justifies distinguishing between patients with and without dementia based on different behavioral, cognitive and functional variables prior to their allocation to a care facility.

\section{Limitations}

A limitation of this review is that the effect sizes could not be calculated for all studies because of missing data. Furthermore, the SCU characteristics [58] as well as the patient groups differed between the reviewed studies. In most of the studies, all patients were suffering from dementia. However, in 2 studies, only 60 and $75 \%$ of the subjects, respectively, suffered from dementia, while the other patients were not cognitively impaired [26, 33]. Eighty-six percent of the studies included patients with different types of dementia, but the remaining 14\% investigated only patients with Alzheimer's disease [35, 38, 41].

The SCU characteristics were often different in the studies reviewed. However, in all studies, the investigators compared different living situations for patients with dementia, one of which was adjusted to their specific needs. A standardization of SCU characteristics will enhance comparability across studies and help to identify specific factors that influence different symptoms of dementia, be it positively or negatively.

Furthermore, the measures and scales (tables 1-4) used to assess behavior, cognition, functional status and quality of life obviously differed between the studies, making the comparison of the effects of living in an SCU or an n-SCU even more challenging. Furthermore, the validity of the measurement scales [59-61] and the sources of the data (patient, e.g. S-MMSE [9]; family, e.g. Neuropsychiatric Inventory [13]; personnel, e.g. ADL [39]) sometimes differed.

Finally, the characteristics of the different SCUs and n-SCUs reviewed are not always similar. The group size, type of training for the personnel and structure of the buildings can differ between the studies reviewed.

The most appropriate research design to study possible relationships between care facilities and the course of dementia and related clinical symptoms would be longitudinal intervention studies. We propose a design in which one group of dementia patients can be assessed before and after moving to an SCU or small-scale, homelike SCU, whereas the other (comparable) group should be assessed during the stay in an SCU or an n-SCU. The use of more specific variables instead of global measurements can provide a better insight into the benefits of specialized care. The development of more sensitive assessment tools is needed due to the bottom effects of conventional assessment instruments in patients with advanced dementia.

As there is no cure for dementia, further studies examining the quality of life of dementia patients, their families and the professional caregivers are needed.

\section{References}

1 Testad I, Aasland A, Aarsland D: The effect of staff training on the use of restraint in dementia: a single-blind randomized controlled trial. Int J Geriatr Psychiatry 2005;20:587-590.

-2 Teri L, Huda P, Gibbons L, Young H, Leynseele J: STAR: a dementia-specific training program for staff in assisted living residences. Gerontologist 2005;45:686-693.

-3 Verbeek H, van Rossum E, Zwakhalen S, Kempen G, Hamers J: Small, homelike care environments for older people with dementia: a literature review. Int Psychogeriatr 2009;21:252-264.

-4 Finnema E, Droës R-M, Ribbe M, van Tilburg W: A review of psychosocial models in psychogeriatrics: implications for care and research. Alzheimer Dis Assoc Disord 2000;14:68-80.

5 Wills W, Trieman N, Leff J: The taps project 40:quality of care provisions for the elderly mentally ill - traditional versus alternative facilities. Int J Geriatr Psychiatry 1998;13:225-234.

6 Weisman G, Kovach C, Cashin S: Differences in dementia services and settings across place types and regions. Am J Alzheimers Dis Other Demen 2004;19:291-298.

7 Cerejeira J, Lagarto L, Mukaetova-Ladinska E: Behavioral and psychological symptoms of dementia. Front Neurol 2012;3:73. 
$\rightarrow 8$ Luo H, Fang X, Liao Y, Elliot A, Zhang X: Associations of special care units and outcomes of residents with dementia: 2004 national nursing home survey. Gerontologist 2004;50:509-518.

9 Nobili A, Piana I, Balossi L, Pasina L, Marina M, Tarantola M, Trevisan S, Riva E, Lucca U, Tettamanti M: Alzheimer special care units compared with traditional nursing home for dementia care. Alzheimer Dis Assoc Disord 2008;22:352-361.

10 De Rooij A, Luijkx K, Declercq A, Emmerink P, Schols J: Quality of life of residents with dementia in traditional versus small-scale long-term care settings: a quasi-experimental study. Int J Nurs Stud 2012;49:931-940.

11 Verbeek H, Zwakhalen S, van Rossum E, Ambergen T, Kempen G, Hamers J: Small-scale, homelike facilities versus regular psychogeriatric nursing home wards: a cross-sectional study into residents' characteristics. BMC Health Serv Res 2010;10:30.

12 Verbeek H, Zwakhalen M, van Rossum E, Ambergen T, Kempen G, Hamers J: Dementia care redesigned: effects of small-scale living facilities on residents, their family caregivers, and staff. J Am Med Dir Assoc 2010;11: 662-670.

13 Te Boekhorst S, Depla M, Lange J de, Pot A, Eefsting J: The effects of group-living homes on older people with dementia: a comparison with traditional nursing home care. Int J Geriatr Psychiatry 2009;24:970-978.

14 Reimer M, Slaughter R, Donaldson C, Currie G, Eliasziw M: Special care facility compared with traditional environments for dementia care: a longitudinal study of quality of life. J Am Geriatr Soc 2004;52:1085-1092.

15 Saxton J, Silverman M, Ricci E, Keane C, Deeley B: Maintenance of mobility in residents of an Alzheimer special care facility. Int Psychogeriatr 1998;10:213-224.

16 Luttenberger K, Donath C, Uter W, Graessel E: Effects of multimodal nondrug therapy on dementia symptoms and need for care in nursing home residents with degenerative dementia: a randomized-controlled study with 6-month follow-up: J Am Geriatr Soc 2012;60:830-840.

17 Kong E, Evans L, Guevara J: Nonpharmacological intervention for agitation in dementia: a systematic review and meta-analysis. Aging Ment Health 2009;13:512-520.

18 Morgan D, Stewart N, D’Arcy K, Werezak L: Evaluating rural nursing home environments: dementia special care versus integrated facilities. Aging Ment Health 2004;8:256-265.

-19 Zeisel J, Silverstein N, Hyde J, Levkoff S, Powell Lawton M, Holmes W: Environmental correlates to behavioral health outcomes in Alzheimer's special care units. Gerontologist 2003;43:697-711.

20 Holmes D, Teresi J, Weiner A, Monaco C, Ronch J, Vickers R: Impacts associated with special care units in longterm care facilities. Gerontologist 1990;30:178-183.

21 Leon J, Marcia O: Effectiveness of special care unit (SCU) placements in reducing physically aggressive behaviours in recently admitted dementia nursing home residents. Am J Alzheimers Dis 1999;14:270-277.

22 Opie J, Rosewarne R, O'Connor D: The efficacy of psychosocial approaches to behaviour disorders in dementia: a systematic literature review. Aust NZ J Psychiatry 1999;33:789-799.

-23 Brawley E: Environmental design for Alzheimer's disease: a quality of life issue. Aging Ment Health 2001; 5:S79-S83.

24 Grant L, Kane J, Stark A: Beyond labels: nursing home care for Alzheimer's disease in and out of special care units. J Am Geriatr Soc 1995;43:569-576.

25 Chappel N, Reid C: Dimensions of care for dementia sufferers in long-term care institutions: are they related to outcomes? J Gerontol B Psychol Sci Soc Sci 2000;55:S234-S244.

-26 Selbaek G, Kirkevold O, Engedal K: Psychiatric and behavioural symptoms and the use of psychotropic medication in special care units and regular units in Norwegian nursing homes. Scand J Caring Sci 2008;22:568573.

27 Ashcraft AS, Owen DC, Feng D: A Comparison of cognitive and functional care differences in four long-term care settings. J Am Med Dir Assoc 2006;7:96-101.

28 Lindesay J, Briggs K, Lawes M MacDonald A, Herzberg J: The domus philosophy: evaluation of a new approach to residential care for the demented elderly. Int J Geriatr Psychiatry 1991;6:727-736.

29 Nakanishi M, Nakashima T, Sawamura K: Quality of life of residents with dementia in a group-living situation. Nihon Koshi Eisei Zasshi 2012;59:3-10.

30 Cohen J: Statistical Power Analysis for the Behavioural Sciences, ed 2. Hillsdale, Lawrence Earlbaum, 1998.

-31 Weyerer S, Schäufele M, Hendlmeier I: Evaluation of special and traditional dementia care in nursing homes: results from a cross sectional study in Germany. Int J Geriatr Psychiatry 2010;25:1159-1167.

-32 Sloane P, Zimmerman S, Gruber-Baldini A, Hebel R, Magaziner J, Konrad T: Health and functional outcomes and health care utilization of persons with dementia in residential care and assisted living facilities: comparison with nursing homes. Gerontologist 2005;45 Spec No I:124-132.

-33 Pekkarinen L, Sinervo T, Elovainio M, Noro A, Finne-Soveri H, Leskinen E: Resident care needs and work stressors in special care units versus non-specialized long term care units. Res Nurs Health 2006;29:465-476.

-34 Frisoni G, Gozzetti A, Bignamini V, Vellas B, Berger A-K, Bianchetti A, Rozzini R, Trabucchi M: Special care units for dementia in nursing homes: a controlled study of effectivness. Arch Gerontol Geriatr 1998;6:215-224.

-35 Swanson E, Maas M, Buckwalter K: Alzheimer's Residents' cognitive and functional measures: special and traditional care unit comparison. Clin Nurs Res 1994;3:27-41.

-36 Kovach C, Weisman G, Chaudhury H, Calkins M: Impacts of a therapeutic environment for dementia care. Am J Alzheimers Dis 1997;12:99-110.

37 Morgan D, Stewart N, D’Arcy C, Forbes D, Lawson J: Work stress and physical assault of nursing aides in rural nursing homes with and without dementia special care units. J Psychiatr Ment Health Nurs 2005;12:347-358. 
-38 Warren S, Janzen W, Andiel-Hett C, Liu L, McKim R, Schalm C: Innovative dementia care: functional status over time of persons with Alzheimer disease in a residential care centre compared to special care units. Dement Geriatr Cogn Disord 2001;12:340-347.

-39 Abrahamson K, Clark D, Perkins A, Arling G: Does cognitive impairment influence quality of life among nursing home residents? Gerontologist 2012;52:632-640.

-40 McAllister C, Silverman M: Community formation and community roles among persons with Alzheimer's disease: a comparative study of experiences in a residential Alzheimer's facility and a traditional nursing home. Qual Health Res 1999;9:65-85.

41 Volicer L, Collard A, Hurley A, Bishop C, Kern D, Karon S: Impact of special care unit for patients with advanced Alzheimer's disease on patients' discomfort and costs. J Am Geriatr Soc 1994;42:597-603.

-42 Nazir A, Arling G, Perkins A, Boustani M: Monitoring quality of care for nursing home residents with behavioral and psychological symptoms related to dementia. J Am Med Dir Soc 2011;12:660-667.

43 Coleman E, Barbaccia J, Croughan-Minihane M: Hospitalization rates in nursing home residents with dementia. J Am Geriatr Soc 1990;38:108-112.

44 Orfaly Cadigan R, Grabowski D, Givens J, Mitchell S: The quality of advanced dementia care in the nursing home: the role of special care units. Med Care 2012;50:856-862.

-45 Phillips C, Sloane M, Hawes C, Koch G, Han J, Spry K, Dunterman G, Williams R: Effects of residence in Alzheimer disease special care units on functional outcomes. JAMA 1997;278:1340-1344.

46 Chafetz P: Behavioral and cognitive outcomes of SCU care. Clin Gerontol 1991;11:19-38

47 Zuidema S, Jonghe J, Verhey F, Koopmans R: Environmental correlates of neuropsychiatric symptoms in nursing home patients with dementia. Int J Geriatr Psychiatry 2010;25:14-22.

48 Smit D, de Lange J, Willemse B, Pot A: The relationship between small-scale care and activity involvement of residents with dementia. Int Psychogeriatr 2012;24:722-732.

-49 Sung H-C, Lee W-l, Li T-l, Watson R: A group music intervention using percussion instruments with familiar music to reduce anxiety and agitation of institutionalized older adults with dementia. Int J Geriatr Psychiatry 2012;27:621-627.

50 Connor D, Ames D, Gardner B, King M: Psychosocial treatments of behaviour symptoms in dementia: a systematic review of reports meeting quality standards. Int Psychogeriatr 2009;21:225-240.

-51 Annerstedt L: Development and consequences of group living in Sweden: a new model of care for the demented elderly. Soc Sci Med 1993;37:1529-1538.

52 Mahoney FI, Barthel DW: Functional evaluation. The Barthel index. Md State Med J 1965;14:61-65.

53 Morris J, Fries B, Morris S: Scaling ADLs within the MDS. J Gerontol A Biol Sci Med Sci 1999;54:M546-M553.

54 Garre-Olmo J, Lopez-Pousa S, Turon-Estrada A, Juvinya D, Ballester D, Vilalta-Franch J: Environmental determinants of quality of life in nursing home residents with severe dementia. J Am Geriatr Soc 2012;60:12301236.

55 Rantakokko M, Iwarsson S, Kauppinen M, Leinonen R, Heikkinen M, Rantanen T: Quality of life and barriers in the urban outdoor environment in old age. J Am Geriatr Soc 2010;58:2154-2159.

$\$ 56$ Cioffi J, Fleming A, Wilkes L, Sinfield M, Le Miere J: The effect of environmental change on residents with dementia: the perceptions of relatives and staff. Dementia 2007;6:215-231.

57 Wiersma E, Pedlar A: The nature of relationships in alternative dementia care environments. Can J Aging 2008; 27:101-108.

58 Verbeek H, van Rossum E, Zwakhalen S, Ambergen T, Kempen G, Hamers J: The effects of small-scale, homelike facilities for older people with dementia on residents, family care givers and staff: design of a longitudinal, quasi-experimental study. BMC Geriatr 2009;9:3.

59 Hall K, Hamilton B, Gordon W, Zasler N: Characteristics and comparisons of functional assessment indices: Disability Rating Scale, Functional Independence Measure, and Functional Assessment Measure. J Head Trauma Rehabil 1993;8:60-74.

60 Reisberg B, Ferris S, de Leon M, Crook T: The Global Deterioration scale for assessment of primary degenerative dementia. Am J Psychiatry 1982;139:1136-1139.

-61 Plutchik R, Conte H, Lieberman M, Bakur M, Grossman J, Lehrman N: Reliability and validity of a scale for assessing the functioning of geriatric patients. J Am Geriatr Soc 1970;18:491-500.

62 Cummings J, Mega M, Gray K, Rosenberg-Thompson S, Carusi D, Gornbein J: The Neuropsychiatric Inventory: comprehensive assessment of psychopathology in dementia. Neurology 1994;44:2308-2314.

63 Zimmerman D: Improving nursing home quality of care through outcome data: MDS quality indicators. Int J Geriatr Psychiatry 2003;18:250-257.

64 De Jonghe J: Factor structure and validity of the Dutch version of the Cohen-Mansfield Agitation Inventory (CMAI-D). J Am Geriatr Soc 1996;44:888-889.

65 Cohen-Mansfield J, Billig N: Agitated behaviors in the elderly: a conceptual review. J Am Geriatr Soc 1986;34: 711-721.

66 Teri L, Truax P, Logsdon R, Uomoto J, Zarit S, Vitaliano P: Assessment of behavioral problems in dementia: the revised memory and behavior problems checklist. Psychol Aging 1992;7:622-631.

67 Morris J, Hawes C, Fries B, Mor V, Katz S, Murphy K, Drugovich M, Friedlob A: Designing the national resident assessment instrument for nursing homes. Gerontologist 1990;30:293-307.

68 Health Care Financing Administration: Long-Term Care Resident Assessment Instrument. User's Manual. Version 2.0. Washington, Department of Health and Human Services, 1995. 
Kok et al.: Special Care Units and Traditional Care in Dementia: Relationship with Behavior, Cognition, Functional Status and Quality of Life - A Review

69 Middleton J, Stewart N, Richardson J: Caregiver distress related to disruptive behaviors on special care units versus traditional long-term care units. J Gerontol Nurs 1999;25:11-19.

70 Alexopoulos G, Abrams R, Young R, Shamoian C: Cornell scale for depression in dementia. Biol Psychiatry $1988 ; 23: 271-284$

-71 Helmes E: Multidimensional Observation Scale for Elderly Subjects (MOSES). Psychopharmacol Bull 1988;24: 733-745.

72 Lawton M, Haitsma van K, Klapper J: Observed affect in nursing home residents with Alzheimer's disease. J Gerontol B Psychol Sci Soc Sci 1996;51:P3-P14.

73 Logsdon R, Teri L: The pleasant events schedule-AD. Psychometric properties and relationship to depression and cognition in Alzheimer's disease patients. Gerontologist 1997;37:40-45.

74 Crocket D, Coval M, Tuokko H, Buree B, Koch W: Multi-Focus Assessment Scale for the Frail Elderly - Revised Form (MAS-R) (Procedure Manual). Vancouver, Department of Psychology, University Hospital, University of British Columbia, 1991.

75 Ray W, Taylor J, Lichtenstein M, Meador K: The nursing home behaviour problem scale. J Gerontol 1992; 47:M9-M16.

76 Rosen W, Mohs R, Davis K: A new rating scale for Alzheimer's disease. Am J Psychiatry 1984;141:1356-1364.

-77 Katona C, Aldridge C: The dexamethasone suppression test and depressive signs in dementia. J Affect Disord 1985;8:83-89.

78 Woods R, Britton P: Clinical Psychology with the Elderly. London, Croom Helm, 1985.

79 Chafetz P, West H: Longitudinal control group evaluation of a special care unit for dementia patients: initial findings. Gerontological Society of America, Washington, 1987.

80 Gurland B, Kuriansky J, Sharpe L, Simon R, Stiller P, Birkett P: The comprehensive assessment and referral evaluation (CARE), rationale, development, and reliability. Int J Aging Hum Dev 1977;8:9-42.

-81 Folstein M, Folstein S, McHugh P: 'Mini-Mental State': a practical method for grading the cognitive state of patients for the clinician. J Psychiatr Res 1975;12:189-198.

82 Morris J, Fries B, Mehr D, Hawes C, Philips C, Mor V, Lipsitz L: MDS Cognitive Performance Scale. J Gerontol 1994;49:M174-M182.

83 Heyman A, Wilkinson W, Hurwitz B, Helms M, Haynes C, Utley C, Gwyther L: Early-onset Alzheimer's disease: clinical predictors of institutionalization and death. Neurology 1987;37:980-984.

84 Goodglass H, Kaplan E: The Assessment of Aphasia and Related Disorders. Philadelphia, Lea \& Febiger, 1972.

85 Gurland B, Kuriansky J, Sharpe L, Simon R, Stiller P, Birckett P: The Comprehensive Assessment and Referral Examination (CARE) - rationale, development and reliability. Int J Ageing Hum Dev 1977;8:9-42.

86 Mattis S: Dementia Rating Scale: Professional Manual. Odessa, Psychological Assessment Resources, 1988.

87 Kahn S, Goldfarb A, Pollack H, Peck A: Brief objective measures of mental status in the aged. Am J Psychiatry 1960;117:326-328.

-88 Engel S, Kiely D, Mitchell S: Satisfaction with end-of-life care for nursing home residents with advanced dementia. J Am Geriatr Soc 2006;54:1567-1572.

89 Teunisse S, Derix M: The interview for deterioration in daily living activities in dementia: agreement between primary and secondary caregivers. Int Psychogeriatr 1997;9(suppl 1):155-162.

$\$ 0$ Reisberg B: Functional assessment staging (FAST). Psychopharmacol Bull 1998;24:653-659.

91 Katz S, Ford A, Moskowitz R, Jackon B, Jaffe M: Studies of illness in the aged. The index of ADL: a standardized measure of biological and psychosocial function. JAMA 1963;185:914-919.

$\$ 92$ Ettema T, Dröes R, de Lange J, Mellenbergh G, Ribbe M: QUALIDEM: development and evaluation of a dementia specific quality of life instrument - validation. Int J Geriatr Psychiatry 2007;22:424-430.

\$3 Kane R, Kling K, Bershadsky B, Kane R, Giles K, Degenholtz H, Liu J, Cutler L: Quality of life measures for nursing home residents. J Gerontol A Biol Sci Med Sci 2003;58:240-248.

94 Rabins P, Kasper J, Kleinman L: Concepts and methods in the development of the ADRQL: an instrument for assessing health-related quality of life in persons with Alzheimer's disease. J Ment Health Aging 1999;5:33-48.

95 Brod M, Steward A, Sands L, Walton P: Conceptualization and measurement of quality of life in dementia: the dementia quality of life instrument (DQoL). Gerontologist 1990;39:25-35.

-96 Lawton M, Weisman G, Sloane P, Norris-Baker C, Calkins M, Zimmerman S: Professional environmental assessment procedure for special care units for elders with dementing illness and its relationship to the therapeutic environment screening schedule. Alzheimer Dis Assoc Disord 2000;14:28-38. 